\title{
Flight Test Evaluation of INS-Aided GPS Tracking Performance under Strong Ionospheric Scintillation
}

\author{
Toshiaki Tsujii, Takeshi Fujiwara, Tetsunari Kubota \\ Institute of Aeronautical Technology, Japan Aerospace Exploration Agency (JAXA) \\ and Yukihiro Kubo \\ Department of Electronics Engineering, Ritsumeikan University, Japan
}

\begin{abstract}
Ionospheric anomaly is one of the major error sources that affect the performance of the GPS (Global Positioning System). In particular, ionospheric scintillation may cause GPS satellite signal loss, and multiple losses can degrade the availability of positioning/navigation services such as GBAS (Ground-Based Augmentation Systems). In order to improve the tracking performance of GPS receivers under scintillation conditions, a tracking loop aided by INS (Inertial Navigation System) has been developed and flight tests to evaluate it were conducted around the island of Ishigaki where ionosphere scintillation frequently occurs. Evaluation of phase tracking performance was carried out off-line using a software-defined GPS receiver which processes stored IF (Intermediate Frequency) data. The use of INS enabled a longer integration time of GPS signals, which increased the signal-to-noise ratio (SNR). As a result, the INS-aided tracking loop achieved continuous phase tracking even under strong scintillation conditions.
\end{abstract}

\section{Introduction}

Although GNSS (Global Navigation Satellite System) is widely used in many kinds of positioning, navigation and timing applications, its accuracy and reliability may not be sufficient under harsh conditions, such as in the presence of ionospheric anomaly. For the safety-of-life applications such as aircraft operations, maintaining high reliability under all conditions is of great importance, so augmentation systems are necessary for critical aircraft operations. In Japan, a satellite-based augmentation system, MSAS (MTSAT Satellite-based Augmentation System), which is compatible with the United States WAAS (Wide Area Augmentation System) and the European EGNOS (European Geostationary Navigation Overlay Service) systems, was developed by the Japan Civil Aviation Bureau and has been available for en-route navigation and non-precision approaches since September 2007. Also, a prototype GBAS for Category I precision approaches has been developed by the Electronic Navigation Research Institute (ENRI). However, even with such augmentation systems GPS availability may be insufficient under conditions such under severe ionospheric scintillation or intentional/unintentional interference (Fig. 1).

While it is well known that the storm-enhanced density
(SED) associated with severe magnetic storms causes a large spatial gradient in ionospheric delay, the effect of plasma bubbles must also be considered in countries at relatively low magnetic latitudes such as Japan, since it is a common phenomenon in such regions and often causes scintillation as well as spatial gradient [1]. Therefore, it is important task to evaluate GPS performance under ionosphere plasma bubbles when introducing a new GPS-dependent safety-of-life technology such as GBAS [2].

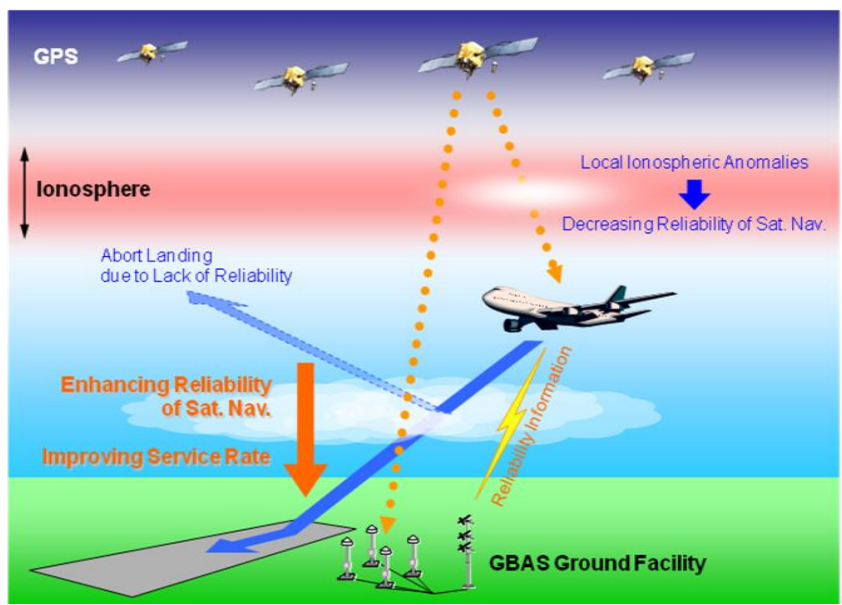

Fig.1 Degraded availability of precision approach due to ionospheric anomalies (conceptual figure)

In this paper, a theoretical estimation of GPS carrier phase error under ionospheric scintillation conditions is first introduced for both ground and airborne receivers. Then, the algorithm of an INS-aided tracking loop for robust tracking under scintillation conditions is introduced. Next, flight tests to gather data to evaluate the tracking performance are described. Analytic results of GPS data acquired under plasma bubbles are then presented, and finally improvement of tracking performance by the INS-aiding technique is demonstrated.

\section{Phase Error Under Scintillation}

Under normal conditions, ground-based GPS receivers are mainly affected by thermal noise and reference clock jitter. In the presence of ionospheric plasma bubbles, receivers are also affected by both amplitude and phase scintillation [3]. The strength of GPS signal intensity scintilla- 
tion is represented by the so-called S4 index expressed by Eq. 1, where GPS signal intensity is given by $I$ and $\langle\cdot\rangle$ represents an operator to take the average of a set of values. Generally, S4 values greater than about 0.7 are regarded as strong scintillation.

$$
S_{4}=\sqrt{\frac{\left\langle I^{2}\right\rangle-\langle I\rangle^{2}}{\langle I\rangle^{2}}}
$$

The standard deviation of carrier phase error caused by receiver thermal noise and amplitude scintillation is given by Eq. 2 [4] and is denoted by 'Scin_Amp' in Fig. 2, in which the S4 index is assumed to be $0.7 . B_{n}$ is the noise bandwidth of the receiver's PLL (phase-locked loop) and $T_{I}$ is the integration time of the input signal. A $T_{I}$ value of $10 \mathrm{msec}$ (milliseconds) is assumed in Fig. 2, although $1 \mathrm{msec}$ is typical for commercial receivers.

$$
\sigma_{\delta \phi, s c i_{-} \text {therm }}{ }^{2}=\frac{B_{n}}{C / N_{0}} \cdot \frac{1}{1-S 4^{2}}\left(1+\frac{1}{2 T_{I} C / N_{0}\left(1-2 \cdot S 4^{2}\right)}\right)
$$

If there is no scintillation ( $\mathrm{S} 4=0)$, the phase error calculated by Eq. 2 is reduced, as shown by the 'Thermal' line in Fig. 2.

The standard deviation of carrier phase error caused by phase scintillation is given by Eq. 3 [4], where $T$ and $p$ are respectively the strength parameter $\left(\operatorname{rad}^{2} / \mathrm{Hz}\right)$ and the dimensionless slope parameter of the phase spectral density under scintillation. Typical values of $T$ and $p(0.5 \mathrm{e}-3$, 2.5) under fairly strong scintillation are used for the calculation of $\sigma_{\delta \phi, s c i n \_p h a s e}$, which is shown as 'Scin_Phase' in Fig 2.

$$
\sigma_{\delta \phi, \text { scin_phase }}^{2}=\frac{\pi T}{2 f_{n}^{p-1} \sin ([p-1] \pi / 4)}
$$

In addition, the phase noise due to clock dynamics for a second-order loop is given in radians ${ }^{2}$ by the following equation [5],

$$
\sigma_{\delta \phi, \text { llock }}^{2}=\left(\frac{2 \pi}{1.8856 B_{n}}\right)^{3} \frac{h_{4} \pi}{2 \sqrt{2}}+\left(\frac{2 \pi}{1.8856 B_{n}}\right)^{2} \frac{h_{3} \pi}{4}+\left(\frac{2 \pi}{1.8856 B_{n}}\right) \frac{h_{2} \pi}{2 \sqrt{2}},
$$

where $h_{2}, h_{3}, h_{4}$ are clock coefficients.

Finally, the total carrier phase error for a second-order PLL is calculated, and is depicted in Fig. 2 where a low elevation satellite $\left(\mathrm{C} / \mathrm{N}_{0}=35.5 \mathrm{~dB}-\mathrm{Hz}\right)$ is assumed and standard TCXO (Temperature Compensated Crystal Oscillator) parameters are applied [5]

The region in which a receiver's PLL can track a signal reliably, assuming the tracking threshold to be 15 degrees (1-sigma value), is shown by the shaded areas in Figs. 2 and 3. For an airborne GPS receiver, vibration-induced jitter and aircraft maneuvers must also to be considered. When the standard power spectral density (PSD) of vibrations given by RTCA (Radio Technical Commission for Aeronautics) DO-160F [6] and the maximum horizontal acceleration $(0.58 \mathrm{~g})$ given by RTCA DO-253C [7] are applied to the PLL model, the computed total phase error for an airborne receiver is shown in Fig. 3. Since the region of stable tracking is much reduced, an airborne receiver is more susceptible to loss of lock than a ground receiver. Therefore, when evaluating GPS performance under plasma bubble conditions, it is of great importance to evaluate actual GPS data obtained from airborne receivers in flight.

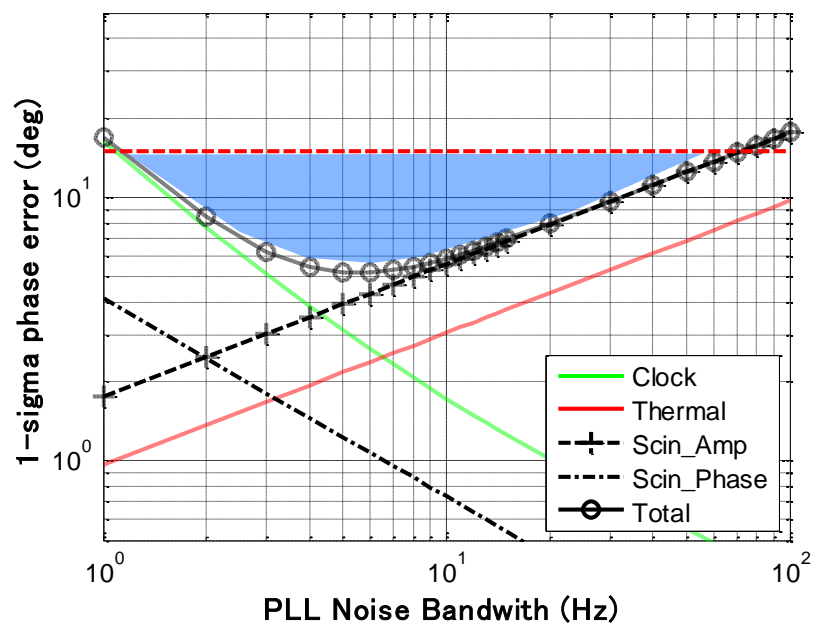

Fig. 2 Carrier phase error of ground receiver

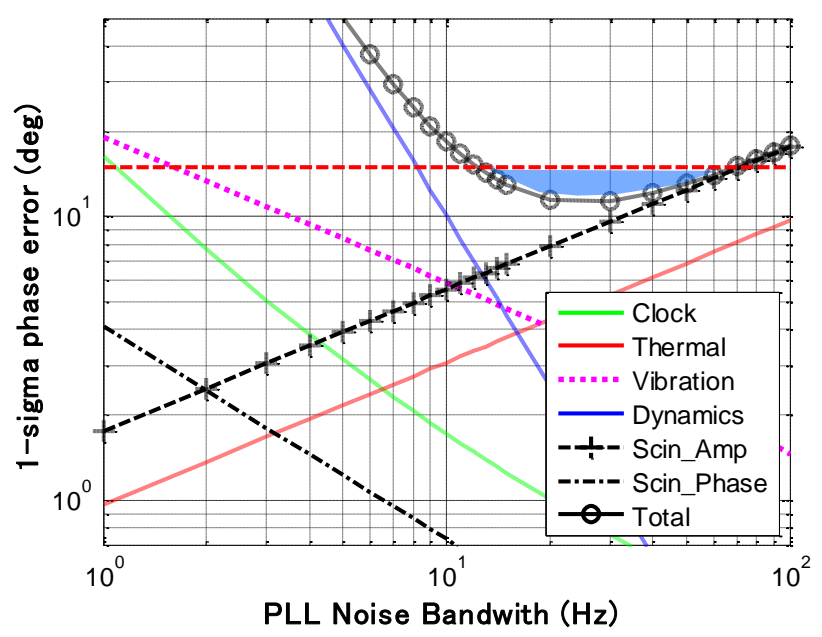

Fig. 3 Carrier phase error of airborne receiver

\section{Doppler-Aided Tracking Loop}

A simple Doppler aiding algorithm in the PLL was developed and the effect of INS aiding was evaluated in a previous work [8]. The Doppler-aided PLL model is shown 
in Fig. 4. $\varphi_{i}$ and $\varphi_{o}$ are the GPS carrier phase input and output signals respectively. A PLL is a feedback loop that controls a carrier numerically-controlled oscillator (NCO) so that the difference between the input phase $\varphi_{i}$ and the output phase $\varphi_{o}$ (a replica of the input phase) becomes zero. A loop filter reduces the noise of the phase error, $\varphi_{i}-\varphi_{o}$, and generates a loop frequency, $f_{P L L 0}$, which is equal to $f_{P L L}$ if the loop is not aided. The model of a conventional non-aided PLL is the same as Fig. 4 except that there is no aiding frequency $\left(f_{A I D}\right)$.

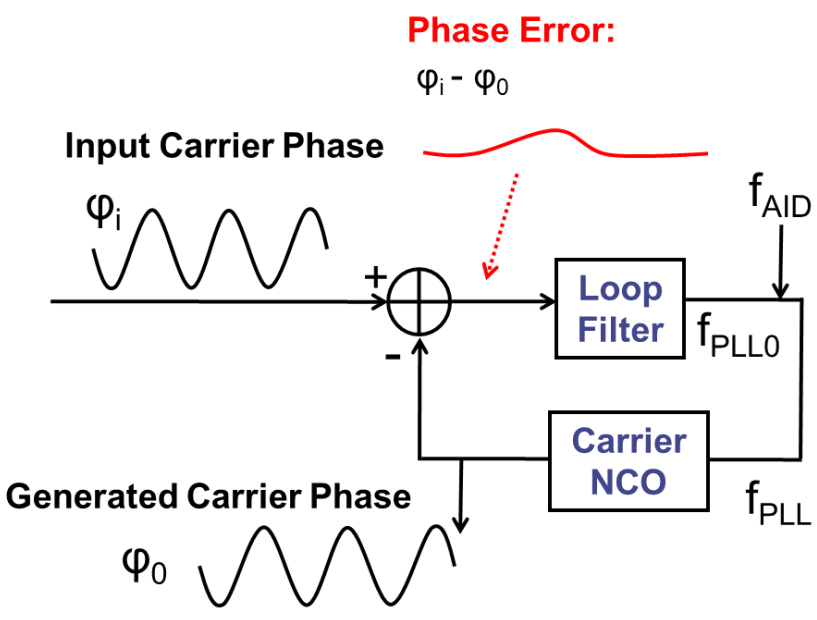

Fig. 4 Doppler-aided PLL model

When an airborne receiver tracks a GPS signal, the carrier frequency is affected by Doppler shift due to the aircraft's speed. Therefore, the PLL's bandwidth should be wider than that of a receiver designed only for ground use, as shown in Figs. 2 and 3. If the Doppler frequency computed using INS information is applied to the PLL, the tracking burden is eased and similar performance to a ground receiver is expected. However, the effects of vibration are not compensated since INS output rates are generally only up to a few hundred Hertz, which is lower than the frequency of aircraft vibrations.

The frequency of the PLL is expressed as:

$$
f_{P L L}=f_{D}+f_{\text {clk }}+f_{\text {noise }}
$$

where $f_{D}, f_{c l k}$ and $f_{\text {noise }}$ are the Doppler, clock, and thermal noise frequencies [5]. If the loop is aided as shown in Fig. 4, the frequency of the PLL can be rewritten as:

$$
f_{P L L}=f_{P L L 0}+f_{A I D}
$$

Three types of aiding frequency $\left(f_{A I D}\right)$ have been tested: Doppler $\left(f_{D}\right)$, delta Doppler $\left(\Delta f_{D}\right)$, and clock frequency $\left(f_{D}+f_{c l k}\right)$ [8]. The Doppler frequency is computed as follows:

$$
f_{D}=\frac{\mathbf{e} \cdot\left(\mathbf{v}_{S}-\mathbf{v}_{R}\right)}{\lambda}
$$

where $\mathbf{v}_{S}, \mathbf{v}_{R}, \mathbf{e}$, and $\lambda$ are the satellite's velocity, the receiver's velocity, the line-of-sight unit vector, and the L1 wavelength, respectively. Delta Doppler is the time difference between the Doppler frequency and the aiding frequency calculated from acceleration measured by an INS instead of velocity as follows:

$$
\Delta f_{D}=-\frac{\mathbf{e} \cdot \mathbf{a}_{R}}{\lambda} \cdot T_{I}
$$

where $\mathbf{a}_{R}$ is the receiver's acceleration. Clock frequency is not obtained from an INS, and has to be estimated. Clock frequency information can be used for the acquisition and tracking of weak signals since it is common for all channels. The clock frequency is computed as follows:

$$
f_{c l k}=\frac{1}{N}\left(\sum_{i=1}^{N}\left(f_{P L L}^{i}-f_{D}^{i}\right)\right)
$$

where the superscript $i$ denotes the $i$ th channel and $N$ is number of tracked channels. Of these three methods, the aiding of Doppler and clock frequency $\left(f_{D}+f_{c l k}\right)$ has shown the best performance [8].

INS aiding can make it possible to lengthen the integration time because rapid changes of Doppler frequency can be compensated by INS information. Fig. 5 shows the phase error caused by scintillation and thermal noise (i.e., $\sigma_{\delta \phi, \text { scin_therm }}$ calculated by Eq. 2) where $\mathrm{C} / \mathrm{N}_{0}$ and $B_{n}$ are $35.5 \mathrm{~dB}-\mathrm{Hz}$ and $10 \mathrm{~Hz}$, respectively. It is clear that phase error is mitigated by increasing the integration time from $1 \mathrm{msec}$ to $20 \mathrm{msec}$. However, further increases would not improve the tracking performance if S4 were less than 0.65. Unfortunately, Eq. 2 is valid only when S4 is less than 0.707 , and there is no theory of phase error estimation under stronger scintillation. Therefore, a direct evaluation of tracking performance was carried out using actual flight data under strong scintillation conditions.

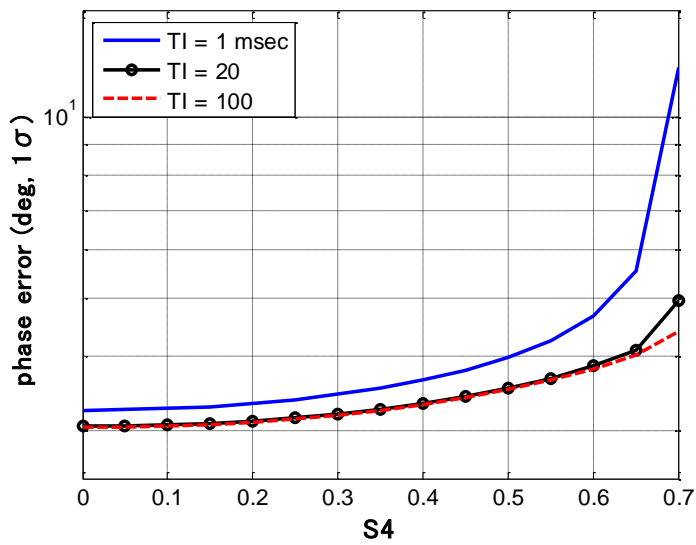

Fig. 5 Phase error vs. S4 with various integration times $\left(\mathrm{C} / \mathrm{N}_{0}=35.5 \mathrm{~dB}-\mathrm{Hz}, B_{n}=10 \mathrm{~Hz}\right)$ 


\section{Flight Tests to Collect Scintillation Data}

The first test was conducted in September 2012 in the area around Ishigaki island, Okinawa prefecture in southwest Japan, and four flights were carried out during the existence of plasma bubbles. By processing the data obtained, the efficacy of the INS-aided tracking loop was validated [9]. The next test was conducted during the period March 19-27, 2013. During this period, plasma bubbles existed on five days $(22,24-27)$ and GPS signal scintillations stronger than in the previous test were observed. The analysis results of the second test are presented in this paper.

\subsection{Ground/Airborne Components}

ENRI operates four GPS observation sites near the former Ishigaki airport. JAXA installed several GNSS receivers at one of ENRI's sites including a Septentrio PolaRxS Pro Ionospheric Scintillation Monitor (ISM), a Javad Delta with a $50 \mathrm{~Hz}$ output option, and a software-based ISM [10] The software-based ISM is of interest since the tracking and scintillation monitoring algorithms can be changed and evaluated by using recorded IF data.

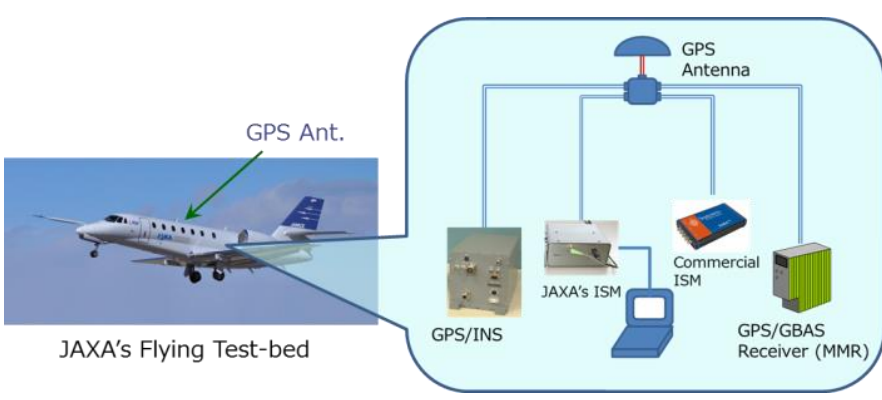

Fig. 6 Research aircraft and onboard components

JAXA's Flying Test-bed (a Cessna Citation Sovereign), shown in Fig. 6, was used for the airborne measurements. It was fitted with the same types of GNSS receivers as on the ground as well as a GPS/GBAS multimode receiver (MMR) and a Rockwell Collins GNLU-930-redlabel. INS data from a GPS and inertial integrated navigation system (GPS/INS) were recorded to be used for the off-line evaluation of the INS-aided tracking loop.

\subsection{Flight Trajectories}

A sample flight trajectory is shown in Fig. 7. Level flight legs of $20 \mathrm{~nm}$ centered on downtown Ishigaki were flown repeatedly. Of particular interest are the flight legs from west to east, since the aircraft's movement might be synchronized with that of plasma bubbles and GNSS signals received by aircraft might be affected for long durations.

Aircraft bank angle and corresponding horizontal acceleration are shown in Fig. 8. Accelerations occasionally exceeding $0.5 \mathrm{~g}$ were achieved during turns of approximately 30 degrees bank. This acceleration is sufficient for the effect of aircraft dynamics on GNSS carrier phase error to be observed during such turns. In addition, pitch reversals were conducted to evaluate the effect of jerk on phase error (Fig. 9). The example shown has jerks larger than the $0.25 \mathrm{~g} / \mathrm{s}$ maximum value for normal maneuvers defined by the GPS airborne equipment Minimum Operational Performance Standards (MOPS) [7].

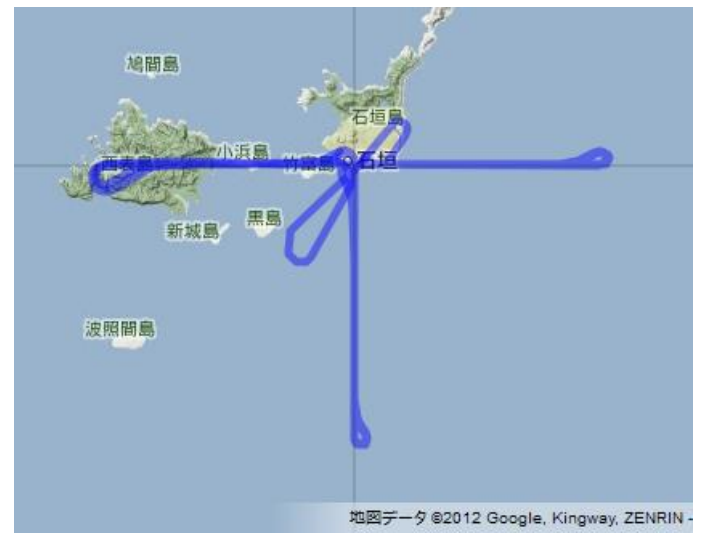

Fig. 7 Sample flight trajectory
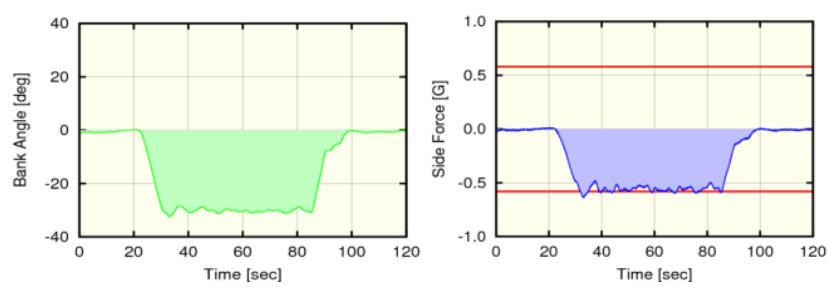

Fig. 8 Aircraft bank angle (left) and horizontal acceleration (right). Red lines indicate $\pm 0.58 \mathrm{~g}$.
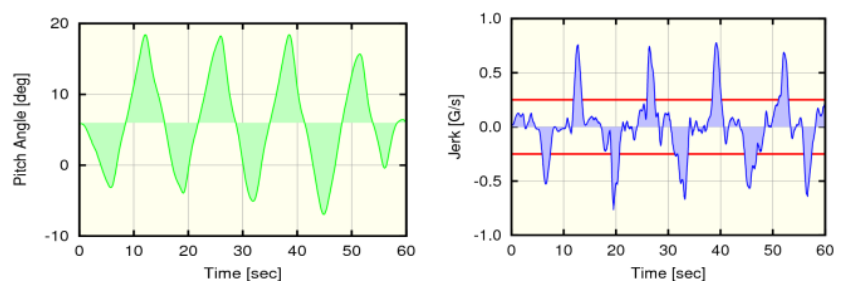

Fig. 9 Aircraft pitch angle (left) and vertical jerk (right). Red lines indicate $\pm 0.25 \mathrm{~g} / \mathrm{s}$

\subsection{Ionospheric Scintillation}

During the March 2013 flight test campaign, the strongest scintillation event was observed on 24 March. Fig. 10 depicts the trajectories of GPS, GLONASS (Global Navigation Satellite System), GALILEO and SBAS (Satellite-Based Augmentation System) satellites from 20:00 to 24:00 local time, with scintillation intensity represented by a color gradient. Very strong scintillations are observed in the southwest, and fairly strong scintillations are seen at high elevation angles even though only satellites up to 30 degrees were affected on other days. Category III GBAS operations would not have possible during this period, since the minimum five satellites required were not available due to signal lock losses and steep gradients of ionospheric delays.

The S4 indices for GPS satellites are shown in Fig. 11 (left). These are the outputs of the airborne PolaRxS Pro 
ISM. The vertical axis indicates satellite PRN numbers (1-32) plus S4 indices. PRN refers to the pseudorandom noise code which is assigned uniquely to each satellite. For example, the S4 index for PRN 11 sometimes exceeded 1.0 during the period 21:00-22:00. Fig. 11 (right) shows the signal lock status, where the vertical axis indicates the value of PRN plus a 'lock flag'. Since the value of lock flag is one when the signal is locked and zero when the signal is lost, occurrences of lock loss can be represented by vertical lines. It is seen that losses of signal lock of PRN 11 correspond to the periods of strong scintillations.

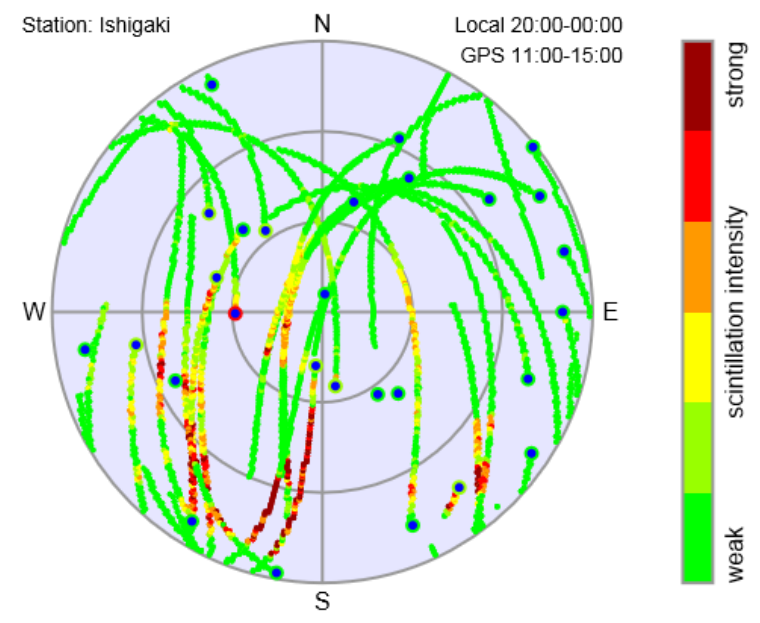

Fig. 10 Satellite trajectories and scintillation intensity (Ground)
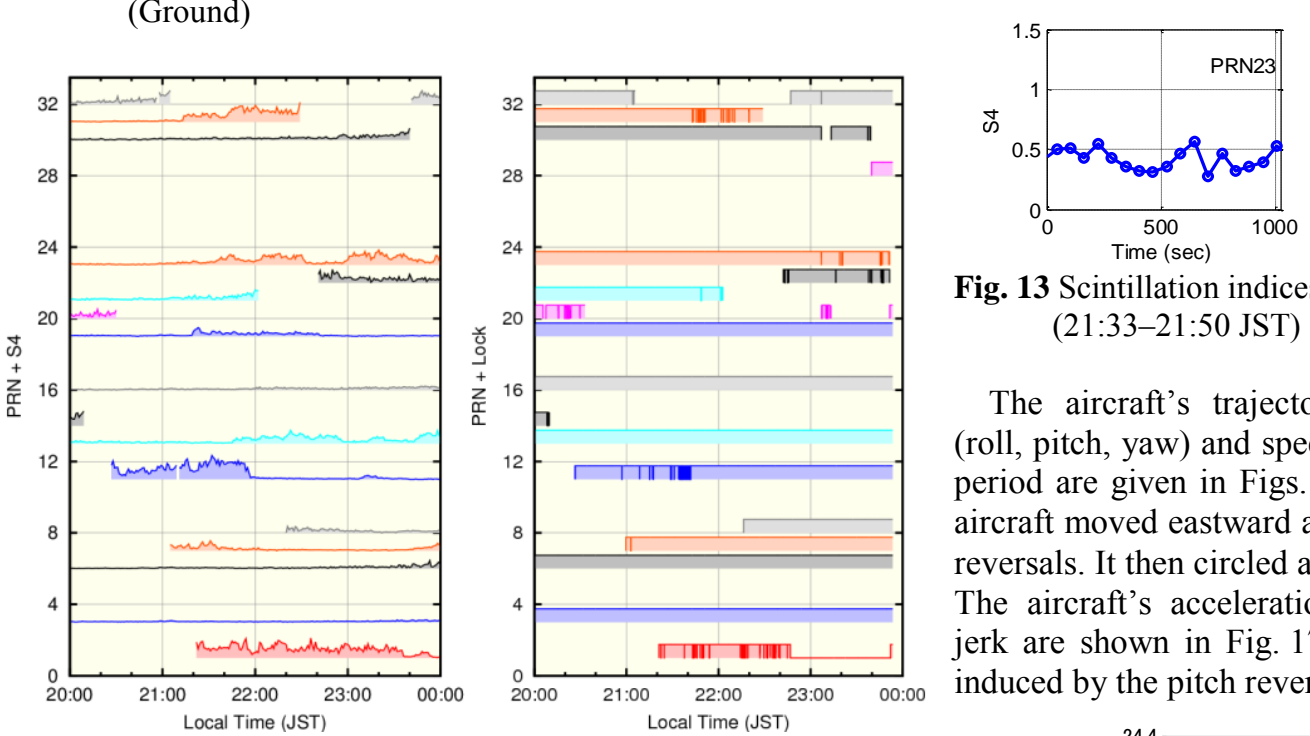

es for these satellites will be evaluated hereafter. The S4 values for PRNs 3 and 6 were small and they did not suffer from scintillation. On the other hand, PRN 23 experienced moderate scintillation with $\mathrm{S} 4$ values up to 0.5 , while PRN 11 suffered from strong scintillation. As seen in Fig. 11, the onboard receiver frequently lost the signal of PRN 11.

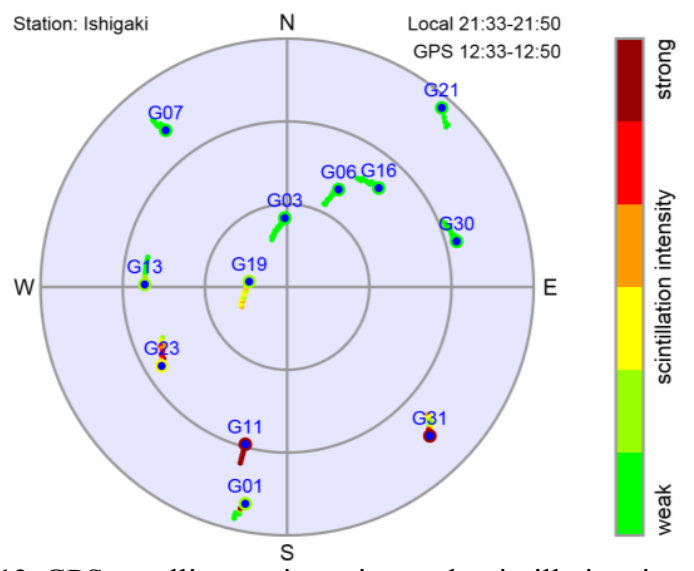

Fig. 12 GPS satellites trajectories and scintillation intensity (21:33-21:50 JST)
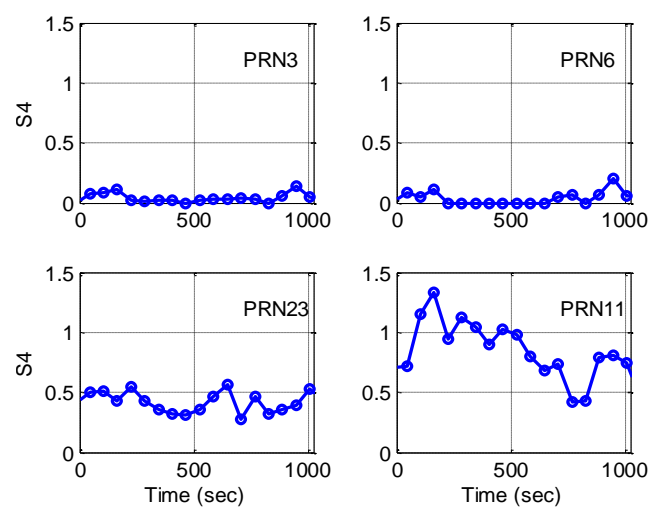

Fig. 13 Scintillation indices (S4) for PRNs 3, 6, 23 and 11 (21:33-21:50 JST)

The aircraft's trajectory (horizontal/vertical), attitude (roll, pitch, yaw) and speed (north, east, down) during that period are given in Figs. 14, 15 and 16, respectively. The aircraft moved eastward after circling, and conducted pitch reversals. It then circled anticlockwise in a 30-degree bank. The aircraft's acceleration (absolute value) and vertical jerk are shown in Fig. 17. Large jerks up to $0.5 \mathrm{~g} / \mathrm{s}$ were induced by the pitch reversals.

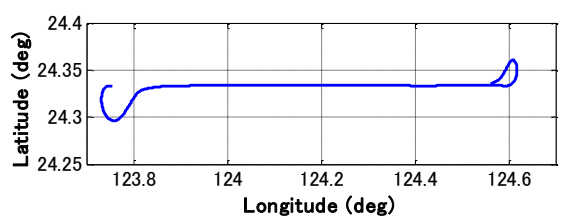

In this section, we focus on the performance of the INS-Aided PLL under strong scintillation conditions during which signal lock might be lost by general Non-Aided receivers. Fig. 12 depicts GPS satellite trajectories and scintillation intensities during the period 21:33-21:50 on March 24. The S4 indices for PRNs 3, 6, 23, and 11 during this period are shown in Fig. 13. The tracking performanc-

\section{Performance of INS-Aided Tracking Loop}

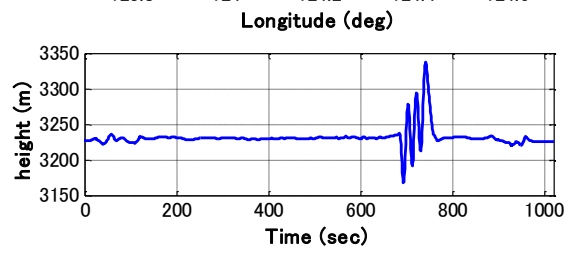

Fig. 14 Aircraft Trajectory (Top: Lat/Lon, Bottom: Height) 


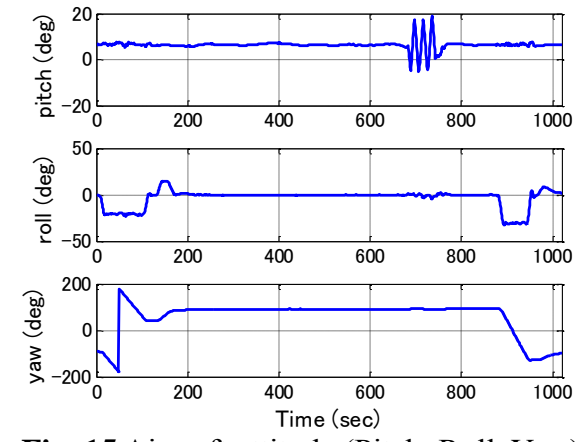

Fig. 15 Aircraft attitude (Pitch, Roll, Yaw)
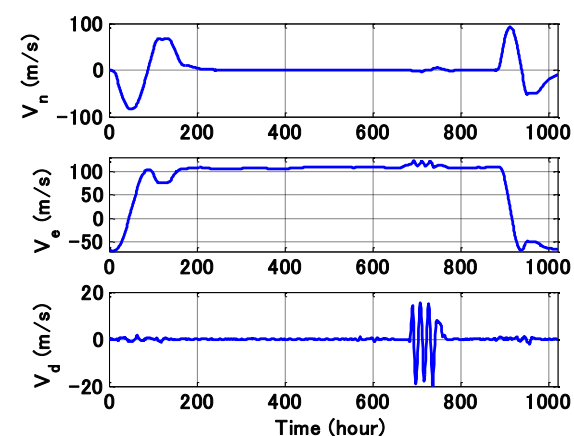

Fig. 16 Aircraft speed (North, East, Down)
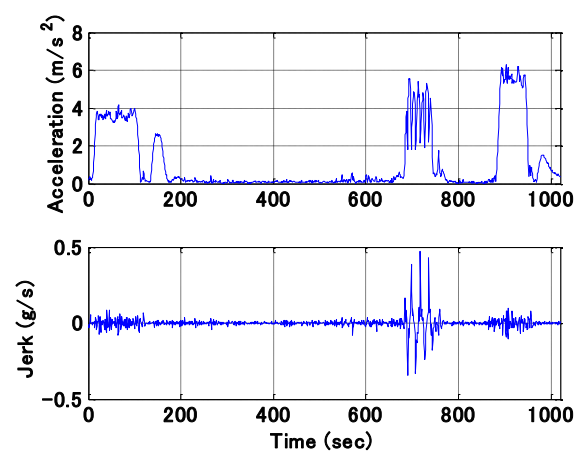

Fig. 17 Aircraft acceleration (absolute value) and jerk (vertical)

The PLL's tracking performance under such scintillation/dynamics conditions are now evaluated. First, the carrier phase tracking errors (discriminator outputs) without aiding for four satellites are shown in Fig. 18. A third-order PLL was applied as in typical dynamic receivers, and the coherent integration time was $1 \mathrm{msec}$.
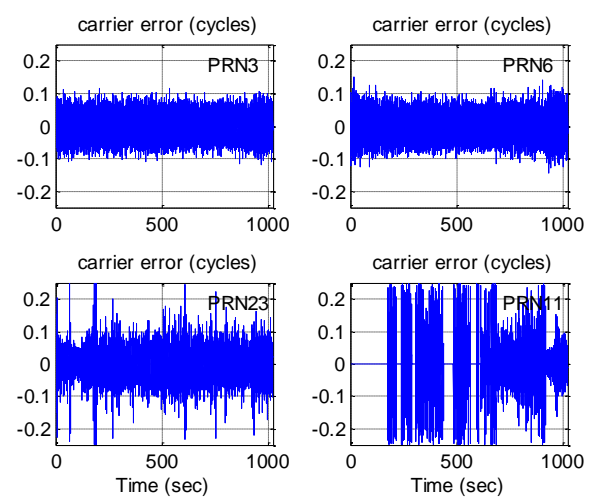

Fig. 18 Carrier tracking error without INS-aiding (Integration time : $1 \mathrm{msec})$
The carrier errors for PRNs 3 and 6 (without scintillation) were stable and up to about 0.1 cycles. This performance is considered to be at the same level as general GPS receivers. On the other hand, several losses of lock occurred for PRN 23, which had moderate scintillation. In the case of PRN 11, continuous tracking was quite difficult, especially when the S4 index exceeded 1.0 (see Fig. 13).

Next, the coherent integration time was increased to $20 \mathrm{msec}$ while the PLL remained non-aided (Fig. 19). Carrier errors were dramatically reduced by increasing the integration time, as expected from Fig. 5. The signal of PRN 23, which was under moderate scintillation, was tracked continuously although was still difficult to track the signal of PRN 11 under strong scintillation. If compared with Fig. 17, some trends related to aircraft dynamics can be seen. While it is well known that a third-order loop does not have steady-state error when acceleration is induced, transient errors as well as the effect of jerk still remain. These trends will be removed by INS-aiding.
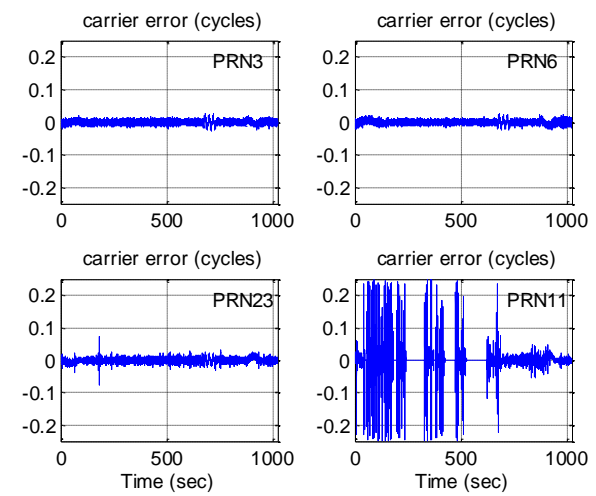

Fig. 19 Carrier tracking error without INS-aiding (Integration time : $20 \mathrm{msec})$

Finally, INS-aiding was applied and the coherent integration time was increased to $100 \mathrm{msec}$. Without INS-aiding, it would not be possible to increase the integration time to such a level. As a result, the carrier phase of PRN 11 under strong scintillation was continuously tracked as shown in Fig. 20, although the phase error was larger than that of other satellites' signals. Regarding other satellites, there were no significant improvements, as expected from Fig. 5.
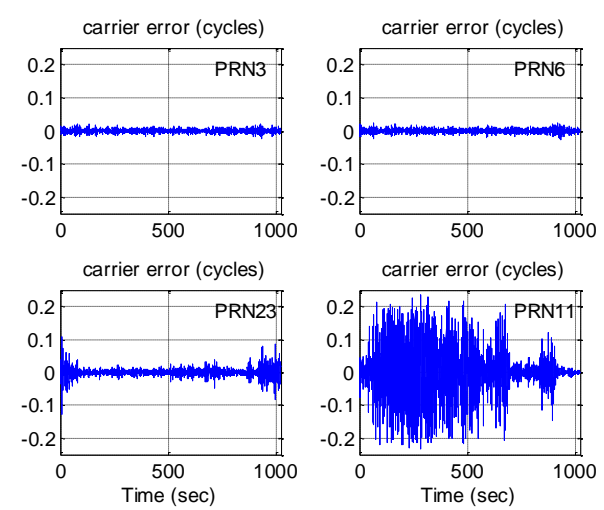

Fig. 20 Carrier tracking error with INS-aiding (Integration time : $100 \mathrm{msec}$ ) 
It was thus demonstrated that GPS carrier phase can be tracked using an INS-aiding technique even under strong scintillation conditions $(S 4 \square 1.0)$. This is a significant improvement because a conventional (non-aided) tracking loop could not track the signal under such conditions as shown in Fig. 11. In previous works, rather short integration times (on the order of 10-20 msec) have been used to track signals affected by scintillation [11, 12], and even shorter integration times (on the order of $1 \mathrm{msec}$ ) have been used to track signals under very strong scintillation [13]. The reason for using short integration times may be that a high feedback rate give better tracking of rapidly fluctuating signals. On the other hand, the authors lengthened the coherent integration time by applying INS-aiding to increase the SNR, and as a consequence robust tracking under strong scintillation was achieved.

\section{Conclusions}

An INS-aided GPS phase tracking loop was developed and its performance was evaluated using flight data acquired under strong scintillation conditions. Although there is no theory to estimate carrier phase error under strong scintillation, the authors assumed that longer integration of GPS signals would mitigate the phase error. Algorithms to increase integration time up to $100 \mathrm{msec}$ by INS-aiding were developed, and off-line analyses using a software-defined GPS receiver were carried out. As a result, it was demonstrated that GPS carrier phase can be tracked using the INS-aiding technique under very strong scintillation conditions ( $S 4 \square 1.0$ ). More detailed analyses will be conducted and the theory to support the results will be investigated in future works.

\section{Acknowledgments}

The authors would like to express gratitude to the Japan Civil Aviation Bureau (JCAB), the Okinawa prefectural government, and Ishigaki city government for their cooperation and support of flight trials. Special thanks go to Dr. Ivan Petrovski of iP-Solutions, Japan, for useful discussions on the tracking loop and scintillation monitor algorithms.

\section{References}

[1] S. Saito: Study of Effects of the Plasma Bubble on GBAS by a Three-Dimensional Ionospheric Delay Model, Proc. of ION GNSS 2009, 2009.

[2] T. Fujiwara, T. Tsujii and T. Kubota: Investigation of Effects of Ionospheric Plasma Bubble on GBAS Availability and its Mitigation by GPS/INS Integration, Proceedings of ION Pacific PNT 2013, pp.959-963, April 22-25, Honolulu, Hawaii, 2013.
[3] M. F. Knight: Ionospheric Scintillation Effects on Global Positioning System Receivers, Ph.D. Thesis, The University of Adelaide, Adelaide, South Australia, December 2000.

[4] R. S. Conker, M. B. El-Arini, C. J. Hegarty, and T. Hsiao, Modeling the Effects of Ionospheric Scintillation on GPS/SBAS Availability, Radio Science, Vol. 38, No. 1, January, 2003.

[5] T.Y Chiou, D. Gebre-Egziabher, T. Walter, and P. Enge: Model Analysis on the Performance for an Inertial Aided FLL-Assisted-PLL Carrier-Tracking Loop in the Presence of Ionospheric Scintillation, Proceedings of the ION National Technical Meeting, San Diego, CA, January 2007.

[6] RTCA: Environmental Conditions and Test Procedures for Airborne Equipment, Section 8, Vibration, RTCA Document No. RTCA/DO-160F, December 8, 2010.

[7] RTCA: Minimum Operational Performance Standards for GPS Local Area Augmentation System Airborne Equipment, RTCA Document No. $R T C A / D O-253 C$, December 16, 2008.

[8] T. Tsujii, T. Fujiwara, Y. Suganuma, H. Tomita and I. Petrovski: Development of INS-Aided GPS Tracking Loop and Flight Test Evaluation, SICE Journal of Control, Measurement, and System Integration, Vol. 4, No. 1, pp. 015-021, 2011

[9] T. Tsujii, T. Fujiwara and T. Kubota: Flight Test Evaluation of INS-Aided GPS Tracking Performance under Equatorial Ionospheric Plasma Bubbles, Proceedings of ION Pacific PNT 2013, pp. 937-945, April 22-25, Honolulu, Hawaii, 2013.

[10] I. Petrovski and T. Tsujii: Digital Satellite Navigation and Geophysics -A Practical Guide with GNSS Signal Simulator and Receiver Laboratory-, Cambridge University Press, 2012.

[11] T. E. Humphreys, M. L. Psiaki, and P. M. Kintner, Jr.: Modeling the effects of ionospheric scintillation on GPS carrier phase tracking, IEEE Transactions on Aerospace and Electronic Systems, vol. 46, no. 4, Oct. 2010.

[12] J. H. Won, B. Eissfelller, T. Pany, and Jón Winkel, Advanced Signal Processing Scheme for GNSS Receivers Under Ionospheric Scintillation, Proceedings of IEEE/ION PLANS 2012, Myrtle Beach, SC, Apr. 24-26, 2012

[13] Macabiau, C. , Deambrogio, L., Barreau, V., Vigneau, W., Valette, J., Artaud, G., Thevenon, P., Ries, L.: Kalman Filter Based Robust GNSS Signal Tracking Algorithm in Presence of Ionospheric Scintillations, Proceedings of the 25th International Technical Meeting of The Satellite Division of the Institute of Navigation (ION GNSS 2012), pp. 3420-3434, Nashville, TN, September 2012. 